\title{
Strates
}

STRATES Matériaux pour la recherche en sciences sociales

$11 \mid 2004$

Jeune recherche, la vitalité d'un laboratoire

\section{Représentations anti-urbaines et aménagement du territoire en Suisse. La ville : perpétuelle mal- aimée?}

Joëlle Salomon Cavin

\section{OpenEdition}

Journals

Édition électronique

URL : http://journals.openedition.org/strates/450

DOI : $10.4000 /$ strates. 450

ISSN : 1777-5442

Éditeur

Laboratoire Ladyss

Édition imprimée

Date de publication : 1 janvier 2004

ISSN : 0768-8067

Référence électronique

Joëlle Salomon Cavin, «Représentations anti-urbaines et aménagement du territoire en Suisse. La ville : perpétuelle mal-aimée? », Strates [En ligne], 11 | 2004, mis en ligne le 14 janvier 2005, consulté le 08 septembre 2020. URL : http://journals.openedition.org/strates/450 ; DOI : https://doi.org/ $10.4000 /$ strates. 450

Ce document a été généré automatiquement le 8 septembre 2020

Tous droits réservés 


\title{
Représentations anti-urbaines et aménagement du territoire en Suisse. La ville : perpétuelle mal- aimée?
}

\author{
Joëlle Salomon Cavin
}

1 Thèse de géographie soutenue à l'École polytechnique fédérale de Lausanne, section Architecture, janvier 2003, sous la direction de Michel Bassand, professeur, (250 p.)

2 L'aménagement du territoire en Suisse est marqué historiquement par l'influence de représentations sociales anti-urbaines qui s'inscrivent dans un contexte général d'hostilité à l'égard de la ville dans ce pays. Un retour sur le devant de la scène de représentations urbanophiles semble s'amorcer depuis la fin des années soixante-dix. Telles ont été les hypothèses générales de cette thèse (Salomon, sous presse).

3 L'analyse du fait urbain en termes de valeurs et de symboles, de même que la détermination de l'inscription culturelle de la pratique de l'aménagement du territoire, constituent désormais des axes de recherche bien explicités dans le cadre de disciplines comme la géographie ou la sociologie (Guérin, Gumuchian, 1985). Par contre, on s'aperçoit que la thématique anti-urbaine ne constitue pas un thème a priori défini par les sciences sociales, même si elle est abordée dans diverses recherches (notamment White, 1962 \& 1977; Treves, 1980 ; Gerosa, Bassand, Racine, 1988; Walter, 1994 ; Marchand, 1999 \& 2001). Cette question apparait pourtant comme une entrée pertinente dans le débat sur la définition du phénomène urbain actuel.

4 La présentation et l'analyse de discours anti-urbains en Suisse sur une longue période, leur confrontation ponctuelle avec d'autres discours nationaux, donnent matière à réflexion concernant les contextes d'émergence, et les contenus des représentations sociales anti-urbaines. Elles montrent très clairement que la société industrielle est inséparable de l'urbanophobie. Alors même que croissent modernité et industrialisation, l'identité nationale, en Suisse comme ailleurs, se construit sur la 
paysannerie et l'idéalisation du monde rural (Thiesse, 2001) et sur la dévalorisation du monde urbain.

5 Les périodes qui vont stimuler l'hostilité à l'égard de la ville correspondent à des moments de turbulences aussi bien économiques, politiques, que sociales. Ce sont, par exemple, les soulèvements politiques contre la domination urbaine à la fin du XVII siècle, les crises économiques, comme celle que traverse l'agriculture suisse à la fin du XIX ${ }^{\mathrm{e}}$ siècle, puis les grands conflits mondiaux.

6 Dans cette étude, les Expositions nationales suisses ont fourni un terrain fertile d'investigation. Ces manifestations périodiques (1896, 1914, 1939, 1964, 2002), singulièrement celle de Genève en 1896, fonctionnent en effet comme d'excellents révélateurs de la place de la ville dans la nation (Salomon, 1998).

7 Une comparaison internationale portant sur la période qui se situe entre les années trente et cinquante révèle que les manifestations anti-urbaines en Suisse ne se distinguent pas de celles observées en France, en Italie ou en Allemagne. Partant, les réflexions en matière d'aménagement du territoire qui émergent en Europe dans les années trente et quarante, et qui précèdent la mise en place d'une politique nationale en ce domaine, sont empreintes d'hostilité à l'égard de la grande ville, non seulement en Suisse mais également dans l'Allemagne nazie, l'Italie fasciste et la France pétainiste.

Des grandes catégories idéal-typiques des représentations anti-urbaines ressortent de cette analyse. Celles-ci ont été intitulées: le village, le sol nourricier, la nature, le paysage, l'anti-centralisation, l'agglomération funeste, la nostalgie, l'absence. Dans cette liste, les figures sans doute les plus évidentes, parce qu'exprimées de façon récurrentes, sont le village et le sol nourricier. Le village, c'est l'antithèse de la société urbaine; c'est l'opposition traditionnelle entre la Gemeindschaft et la Gesellschaft (Tönnies, 1877) : un idéal à échelle humaine, une façon de vivre ensemble à proximité les uns des autres, un univers tranquille de relations personnalisées. On y est solidaire et on y a le sens de la communauté. Le sol nourricier, c'est la ville stérilisatrice à la fois de la terre et des familles. La campagne nourrit la nation et lui donne des hommes, alors que la ville détruit la terre et les individus eux-mêmes. C'est la ville " gouffre de l'espèce humaine » dont parle Rousseau (1761).

9 Une étude détaillée du cas vaudois et lausannois confirme l'hypothèse de l'influence des représentations anti-urbaines sur l'aménagement du territoire au niveau local et cantonal. Si les tentatives de planification concertée de la région lausannoise sont le signe d'une conscience précoce chez les professionnels de la ville d'une nécessaire vision globale de l'aménagement urbain, leur échec est directement imputable à l'hostilité des responsables politiques à l'égard de la ville-centre. Les représentations négatives de la ville constituent un élément récurrent de la politique d'aménagement du territoire menée dans le canton de Vaud depuis les années quarante. Partant, l'aménagement cantonal a surtout tendu à freiner et circonscrire le développement urbain dans le canton plutôt qu'à le gérer.

10 Amorcé à partir des années soixante-dix dans la recherche (notamment Bassand, Joye, Schuler, 1988), un retour sur le devant de la scène de représentations positives de la ville suisse se confirme dans les années quatre-vingt-dix au niveau de la politique fédérale d'aménagement du territoire et dans les contextes cantonal et local étudiés.

11 En 2002, les agglomérations deviennent la priorité de la nouvelle politique de développement territorial de la Confédération (Conseil fédéral, 2001). 
endant, le regard porté sur le territoire suisse est aujourd'hui loin d'être univoque d'un côté, l'image d'une Suisse urbaine, voire métropolitaine, projetée par les milieux scientifiques et professionnels, et de l'autre, la projection diffuse d'un pays demeuré peu urbain et que l'on imagine encore préservé des maux contemporains qui accompagnent l'urbanisation. La sensibilisation de la population et des acteurs politiques à la réalité urbaine actuelle constitue désormais le nouvel enjeu de la politique d'aménagement du territoire en Suisse.

\section{BIBLIOGRAPHIE}

Bassand M., Joye D., Schuler M., Les enjeux de l'urbanisation en Suisse : Agglomerationsprobleme in der Schweiz, Peter Lang, Berne, 1988.

Conseil fédéral, Politique des agglomérations de la Confédération, Berne, 2001.

Gerosa P.G., Bassand M., Racine J.-B., «L'urbain et l'idéologie », in Les enjeux de l'urbanisation en Suisse : Agglomerations-probleme in der Schweiz, Peter Lang, Berne, 1988, p. 115-133.

Guérin J.-P., Gumuchian H. (ed.), Les représentations en actes, actes du colloque de Lescheraines, Grenoble, Institut de géographie alpine, 1985.

Marchand B. « Nationalsozialismus und Grossstadtfeind-schaft », Die alte Stadt, 1, 1999, p. 39-50.

Marchand B. « La haine de la ville : Paris et le désert français de Jean-François Gravier », L'information géographique, 3, 2001, p. 234-253.

Rousseau J.-J., « Émile ou de L'éducation », 1761, in Euvres complètes, Gallimard, Paris, 1964.

Salomon J., "Les Suisses et la ville, un rapport ambigu illustré par les expositions nationales », Acta Geographica, n 114, 1998, p. 33-48.

Salomon J., « Modes d'habiter, modes de planifier en Suisse : la ville mal aimée », in Actes du colloque Nouvelles ruralités, nouvelles urbanités en Europe, Peter Lang ed. (sous presse).

Thiesse A.-M., La création des identités nationales, Seuil, Paris, 2001.

Tönnies F., Communauté et société : catégories fondamentales de la sociologie pure, Retz-CEPL, Paris, 1977.

Treves A., " The anti-urban policy of fascism and century of resistance to industrial urbanization in Italy », International Journal of urban and Regional Research, 4, 1980, p. 470-484.

Walter F., «Les implications idéologiques de la problématique urbaine », La Suisse urbaine, 1750-1950, éditions Zoé, Carouge-Genève, 1994, p. 422-440

White M., " Two Stages in the Critique of the American City », in O. Handlin, J. Burchard, The Historian and the City, Cambridge, MIT Press, 1977.

White M., White L., The Intellectual Versus the City: from Thomas Jefferson to Franck Lloyd Wright, Harvard University Press, Cambridge, 1962.

Strates, $11 \mid 2004$ 
INDEX

Index géographique : Suisse

Mots-clés : Ville 